\title{
A POSSIBLE UPGRADE FOR THE SRS RF SYSTEM
}

\author{
D M Dykes, P. A. McIntosh, A J Moss, CLRC Daresbury Laboratory, Warrington, WA4 4AD, UK
}

\begin{abstract}
The world's first dedicated source for synchrotron radiation, the Synchrotron Radiation Source (SRS) at Daresbury, UK is now 20 years old. During its operational lifetime, the control and stability of the electron and photon beams have been significantly improved, to ensure continued reliable operation, in order to necessitate various lattice and insertion device (ID) upgrades [1][2]. The SRS currently utilises four $500 \mathrm{MHz}$ accelerating cavities, which can provide a maximum of $2.1 \mathrm{MV}$ accelerating voltage, equating to $\sim 300 \mathrm{~mA}$ of stored beam current at $2 \mathrm{GeV}$. To operate the SRS at this beam current level routinely for users, would undoubtedly be very difficult due to cavity induced beam instabilities. As a possible way of upgrading the SRS to operate at these beam current levels and beyond, it is proposed to replace the existing SRS RF cavities with two new higher order mode (HOM) damped cavities. This would enable beam currents up to 1 Ampere to be stored both reliably and with a higher degree of stability. The availability of two additional straight sections would then offer the possibility of introducing two high field MPW devices. This paper details the new cavity design, highlighting the associated RF system modifications that would become necessary.
\end{abstract}

\section{INTRODUCTION}

Multi-bunch instability limits have been previously observed on the SRS and have so far been avoided by careful adjustment of the cavity temperatures to sustain normal operations [3]. In order to reliably achieve beam currents $>250 \mathrm{~mA}$ on the SRS, experience has shown that the cavity temperature stability is critical and the temperature range over which control of the multibunch instabilities is maintained, is severely restricted the larger the stored beam currents.

The total RF power $\left(P_{T}\right)$ required on an accelerator is derived from:

$$
P_{T}=P_{C}+P_{b}+P_{P}
$$

$P_{C}$ is the cavity power necessary to accelerate the beam and is evaluated from; $P_{C}=\left(U_{o} q\right)^{2} / 2 R_{s}$, where $q$ is the overvoltage factor, $U_{o}$ is the energy loss/turn (KeV/turn) and $R_{s}$ is the cavity shunt impedance (M $\Omega$ ). $P_{b}$ is the beam power required to replace the energy lost to synchrotron radiation and is derived from $P_{b}=U_{o} I_{b}$, where $I_{b}$ is the stored beam current. $P_{P}$ is the power required to overcome any parasitic losses and from practical measurements on the SRS, equates to $\sim 10 \mathrm{~kW}$ at $250 \mathrm{~mA}$. Table 1 shows that for the $321 \mathrm{keV}$ energy loss per turn on the SRS, if the existing RF cavities are employed, $475 \mathrm{~kW}$ of $\mathrm{RF}$ power would be required to achieve 1 Ampere. This would necessitate an overvoltage change from $q=3.75$ to $q=5.5$, for system stability reasons, which could be accommodated in the existing cavity feeder design.

Table 1. RF Power Needed for Existing SRS Cavities.

\begin{tabular}{|c|c|c|c|c|c|c|c|}
\hline \multicolumn{7}{|c|}{ RF Power Components $(\mathrm{kW})$} \\
\hline \multicolumn{4}{|c|}{$250 \mathrm{~mA}(q=3.75)$} & \multicolumn{4}{|c|}{$1 \mathrm{~A}(q=5.5)$} \\
\hline$P_{C}$ & $P_{b}$ & $P_{P}$ & $\boldsymbol{P}_{\boldsymbol{T}}$ & $P_{C}$ & $P_{b}$ & $P_{P}$ & $\boldsymbol{P}_{\boldsymbol{T}}$ \\
\hline 51 & 80.2 & 10 & $\mathbf{1 4 1 . 2}$ & 112.5 & 321 & 40 & $\mathbf{4 7 5}$ \\
\hline
\end{tabular}

The total RF power figures quoted in Table 1 are for a critically matched RF system, whereby all cavities are resistively tuned, this however is not easily achieved on the SRS as its energy is ramped and the beam loading in the cavities can vary dramatically under these conditions. Therefore the cavities are normally inductively detuned to avoid Robinson instability and consequently more RF power is required to account for the additional reflected power. This can amount to $2-3 \mathrm{~kW}$ of additional RF power required per cavity (at $250 \mathrm{~mA}$ ), which means that the total RF power $P_{T}$ at 1 A would rise to $\sim 490 \mathrm{~kW}$.

As a way of overcoming the multibunch instabilities and also improving RF power transfer efficiency, a replacement of the existing RF cavities with a new, higher efficiency accelerating structure has been investigated. This new optimised cavity design could inherently damp the HOM impedances whilst also enabling a larger RF input power; large enough to necessitate a requirement for only two cavities instead of the original compliment of four. This would then release a further two ID straights, for installation of devices which would benefit from the increased photon flux available.

\section{THE NEW RF CAVITY DESIGN}

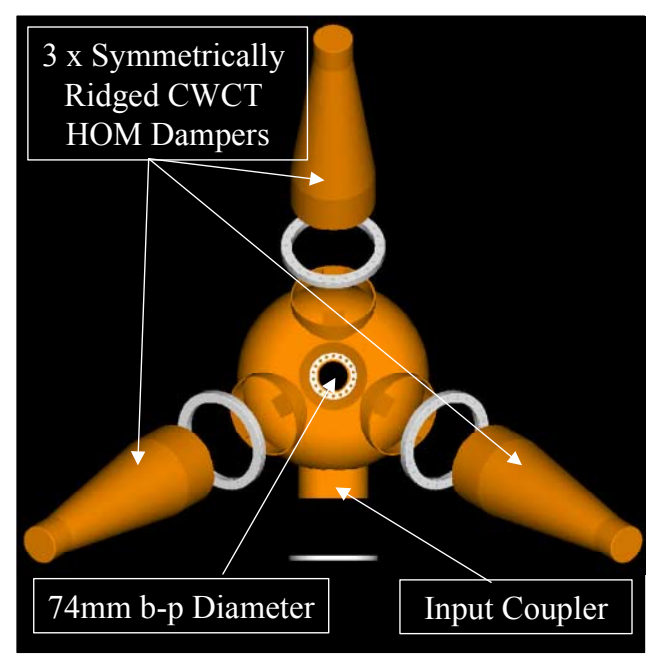

Figure 1. Spherical EU Cavity Design Assembly. 
As part of an EU collaborative team, Daresbury Laboratory has already led the design of a spherical, reentrant cavity, operating at $500 \mathrm{MHz}$, which incorporates 3 equispaced ridged waveguide tapers, with a circular waveguide to coaxial transition (CWCT) to couple out the HOM power to external HOM loads. Figure 1 shows a layout for the spherical EU cavity design incorporating the 3 CWCT damping waveguides along with a longitudinally offset, aperture input coupler, which also provides additional narrowband HOM damping.

This cavity design employs a $74 \mathrm{~mm}$ beam-pipe diameter to primarily enhance the fundamental mode shunt impedance with an $\mathrm{R}_{\mathrm{s}}=4.7 \mathrm{M} \Omega$, a consequence of which is to also enhance the HOM shunt impedances. The magnitude of these limiting impedance characteristics, calculated using MAFIA [4] are discussed in more detail elsewhere [5]. They do however highlight excellent HOM damping characteristics, when compared to the current generation of normal conducting, HOM damped cavities, such as those employed on the PEP-II [6] and DAФNE [7] accelerators.

Table 2. SRS Machine Parameters

\begin{tabular}{|l|c|c|}
\hline Parameter & Symbol & Value \\
\hline RF Frequency (MHz) & $f_{R F}$ & 499.7 \\
\hline Energy (GeV) & $E$ & 2 \\
\hline Revolution Frequency (MHz) & $f_{\text {rev }}$ & 3.123 \\
\hline Synchrotron Tune & $Q_{s}$ & 0.01748 \\
\hline Momentum Compaction & $\alpha$ & 0.0288 \\
\hline Longitudinal Damping Time (ms) & $\tau_{s}$ & 1.988 \\
\hline Transverse Damping Time x,y (ms) & $\tau_{x, y}$ & $4.2,4.0$ \\
\hline Beta x,y (m) & $\beta_{x, y}$ & $5.85,1.93$ \\
\hline Number of Cavities & $n_{c a v}$ & 4 \\
\hline
\end{tabular}

For the SRS machine parameters as shown in Table 2, longitudinal (Equation 2) and transverse (Equation 3) multibunch instability beam current thresholds have been computed for this EU cavity design of Figure 1 and predict thresholds of: $I_{t h}^{\prime \prime}=473 \mathrm{~mA}$ and $I_{t h}^{\perp}=553 \mathrm{~mA}$ respectively.

$$
\begin{aligned}
\text { Longitudinal, } I_{t h}^{\prime \prime} & =\frac{2 E Q_{s}}{\alpha \tau_{s} R_{H O M}^{\prime \prime} f_{H O M}^{\prime \prime} n_{c a v}} \\
\text { Transverse, } I_{t h}^{\perp} & =\frac{2 E}{\beta_{x, y} \tau_{x, y} R_{H O M}^{\perp} n_{c a v} f_{r e v}}
\end{aligned}
$$

The figure of merit for longitudinal instability performance for a particular cavity solution, from Equation 2 is the maximum impedance-frequency product in the bandwidth below beam-pipe cut-off $\left(f_{c}^{T M 01}=3.1 \mathrm{GHz}\right)$ and above CWCT cut-off $\left(f_{c}^{C W C T}=650 \mathrm{MHz}\right) \quad$ frequencies. The corresponding limitation for the transverse plane is purely the maximum transverse HOM impedance as there is no inverse frequency dependence (see Equation 3). The HOM damping for this cavity design is such that for beam currents up to $500 \mathrm{~mA}$ operation on the SRS, this would be a viable cavity solution. For $1 \mathrm{~A}$ operation however, its HOM damping characteristics require some improvement, to avoid the potential for mutlibunch instabilities.

An adaptation of the EU cavity design has therefore been performed to try and improve the cavity HOM damping characteristics. Figure 2 illustrates the spherical EU cavity geometry and the associated modifications that have been implemented.

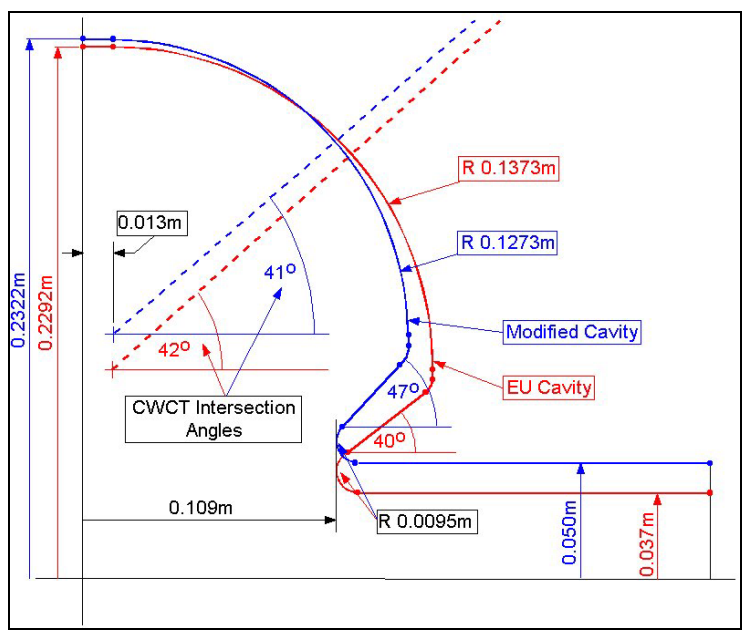

Figure 2. EU Cavity and Modified Cavity Geometries.

By relaxing the beam-pipe dimension from $74 \mathrm{~mm}$ diameter for the EU cavity design, to $100 \mathrm{~mm}$ diameter and re-correcting for $500 \mathrm{MHz}$ operating frequency, a reduction in the longitudinal AND transverse HOM impedances are observed (see Figure 3).

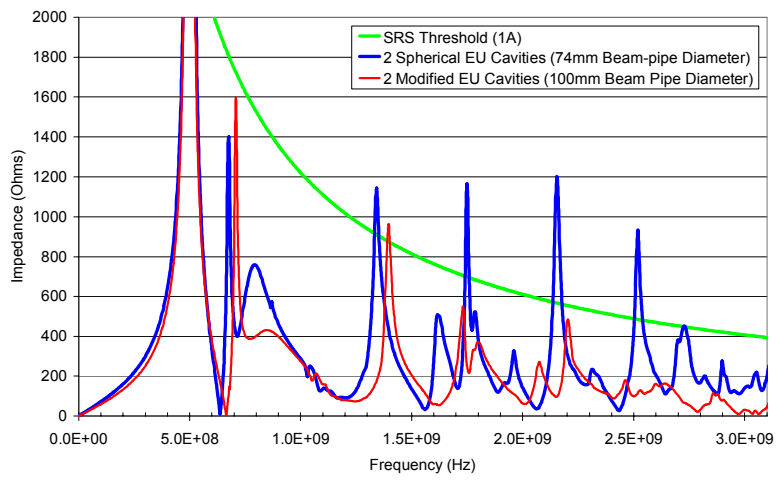

Figure 3. Cavity Longitudinal HOM Spectrum.

This can be seen by evaluation of the HOM impedance spectra for the two cavity geometries and comparing with the machine impedance at which, the HOM growth rates equal the machine damping times. This effectively defines the point at which the onset of multibunch instabilities occurs. Figure 3 shows the longitudinal impedance spectrum of the spherical EU cavity with a $74 \mathrm{~mm}$ beampipe and that of a modified design incorporating a $100 \mathrm{~mm}$ beam-pipe, compared with these is the impedance threshold for the SRS at 1A.

The technique employed for the characterisation of 
such HOM damped structures has been detailed elsewhere and uses a MAFIA time-domain excitation technique to compute the longitudinal and transverse wakefield responses, which are Fast Fourier Transformed (FFT) and normalised by the gaussian bunch excitation signal [8].

The HOM performance figures of merit for the two geometry options are shown in Table 3 along with the corresponding beam current thresholds predicted when two of each cavity type are applied on the SRS.

Table 3. Beam Current Thresholds.

\begin{tabular}{|l|c|c|}
\cline { 2 - 3 } \multicolumn{1}{c|}{} & $\begin{array}{c}\text { EU Cavity } \\
(74 \mathrm{~mm} \text { b-p })\end{array}$ & $\begin{array}{c}\text { Modified } \\
(100 \mathrm{~mm} \text { b-p })\end{array}$ \\
\hline Max $R_{H O M}^{\prime \prime} f_{H O M}^{\prime \prime}(\mathrm{Hz} . \Omega)$ & $1.29 \mathrm{e} 12$ & $6.72 \mathrm{e} 11$ \\
\hline Long. Threshold $I_{t h}^{\prime \prime}(\mathbf{m A})$ & $\mathbf{4 7 3}$ & $\mathbf{9 0 8}$ \\
\hline Max $R_{H O M}^{\perp}(\mathrm{k} \Omega / \mathrm{m})$ & 46.9 & 20.7 \\
\hline Trans. Threshold $I_{t h}^{\perp} \mathbf{( m A )}$ & $\mathbf{5 5 3}$ & $\mathbf{1 2 5 0}$ \\
\hline
\end{tabular}

The improvement in the longitudinal performance is clear, an almost doubling of the predicted beam current threshold limit from $I_{t h}^{\prime \prime}=473$ to $908 \mathrm{~mA}$. The increased beam pipe dimension has also benefited the transverse HOM impedances significantly as shown in Figure 3, with a beam current threshold improvement from $I_{\text {th }}^{\perp}=553$ to $1250 \mathrm{~mA}$.

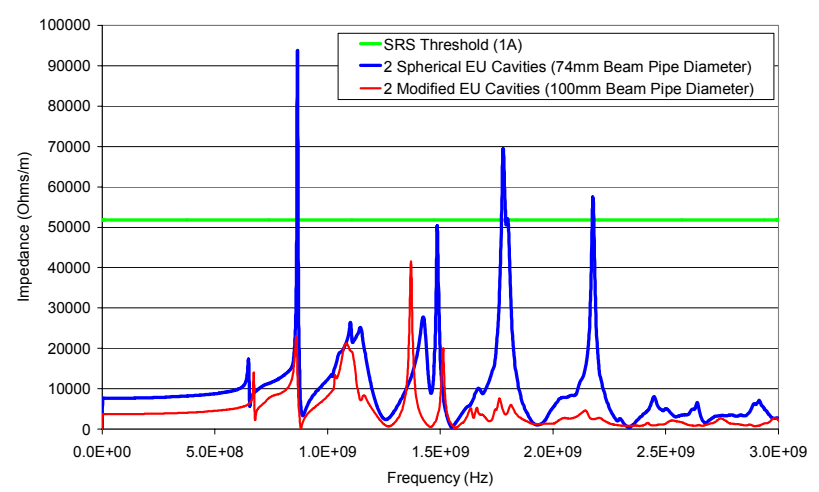

Figure 3. Cavity Transverse HOM Spectrum.

The accelerating efficiency or fundamental mode shunt impedance has been estimated for this modified cavity geometry as $R_{s}=4.3 \mathrm{M} \Omega$. This equates to a $9 \%$ reduction in $R_{s}$ compared to the original EU cavity design of $4.7 \mathrm{M} \Omega$. Although more RF power would be required to generate the necessary accelerating voltage, the increased HOM damping for this modified cavity design more than compensates for its marginally reduced efficiency, plus the fact that it is already $20 \%$ more efficient than the current non-HOM damped SRS cavities.

\section{CAVITY APPLICATION ON THE SRS}

This new cavity design would be capable of providing an acceleration voltage of $850 \mathrm{kV} /$ cell. If two cavities were employed in the SRS, requiring a momentum aperture voltage of $1.2 \mathrm{MV}$, the power required to generate this voltage would be $84 \mathrm{~kW}$.

Table 4. RF Requirements For New RF Cavities.

\begin{tabular}{|c|c|c|c|}
\hline \multicolumn{4}{|c|}{ Modified Cavity RF Power at $1 \mathrm{~A}(q=3.75)$} \\
\hline $\mathrm{P}_{\mathrm{C}}$ & $\mathrm{P}_{\mathrm{B}}$ & $\mathrm{P}_{\mathrm{P}}$ & $\mathbf{P}_{\mathrm{T}}$ \\
\hline 84 & 321 & 40 & $\mathbf{4 3 0}$ \\
\hline
\end{tabular}

The new configuration of only 2 cavities could either be fed by a single $500 \mathrm{~kW}$ klystron via a single Magic-Tee and isolation transformer, or else by $2 \times 250 \mathrm{~kW}$ klystrons each cavity being fed by a single klystron. The latter option is a more cost-effective solution on the SRS, as $2 \mathrm{x}$ $250 \mathrm{~kW}$ klystrons are already available as part of a "hot" standby amplifier system, should the operational klystron fail [9].

\section{CONCLUSIONS}

A possible upgrade of the SRS RF system to enable beam currents approaching $1 \mathrm{~A}$ to be more reliably achieved with a higher degree of stability has been identified. A new HOM damped cavity has been designed, adapting the already excellent performance of a cavity developed by Daresbury Laboratory as part of an EU collaboration. The predicted performance of this new cavity design suggests that stable longitudinal and transverse operation could be expected on the SRS approaching this $1 \mathrm{~A}$ upgrade goal.

\section{REFERENCES}

[1] J A Clarke et al, "High Resolution Stabilisation of Vertical Photon Beam Position in SRS Beamlines", EPAC'94, London 1994, pp 125-127.

[2] J A Clarke et al, "Upgrading the Daresbury SRS with Additional Insertion Devices and its Implications for the Storage Ring Layout", EPAC'96, Sitges, 1996, pp623625.

[3] P A McIntosh et al, "HOM Induced Longitudinal Coupled Bunch Instabilities at the SRS”, EPAC'96, Sitges, 1996, pp1135-1137.

[4] "MAFIA Manual Version 4.00", CST, Darmstadt, Germany.

[5] F Marhauser et al, "HOM Damped 500MHz Cavity Design for $3^{\text {rd }}$ Generation SR Sources", these proceedings.

[6] R A Rimmer et al, "Updated Impedance Estimate of the PEP-II RF Cavity”, EPAC'96, Sitges, 1996, pp20352037.

[7] R Boni et al, "High Power Test of the Waveguide Loaded RF Cavity for the Frascati $\Phi$-Factory Main Rings", EPAC'96, Sitges, 1996, pp1979-1981.

[8] Ch Ng et al, "Impedance Spectrum for the PEP-II RF Cavity", PAC'95, Dallas, 1995, pp1744-1746.

[9] P A McIntosh et al, "Recommissioning of the RF System After the SRS Upgrade", PAC'99, New York, 1999, pp1025-1027. 